\title{
Make What You Desire: An Interactive Blending of the Digital and the Analogue
}

\author{
Denise Allan \\ Edinburgh Napier University \\ 10 Colinton Road, Edinburgh \\ denise.allan@napier.ac.uk
}

\begin{abstract}
3D printing technology has the potential to democratize the manufacturing process. 3D printers have become more affordable so almost anyone can access them either at home, work or a local Fablab. Now that this access is becoming more widespread, it is interesting and perhaps useful to find out what people are making, or at least what they want to make. This short, work in progress paper will illustrate how the author is attempting to find out what people want to make, while at the same time making 3D printing more visible where it might otherwise not be found.
\end{abstract}

3D printing, Design, Manufacturing, Democratization, 3D scanning

\section{INTRODUCTION}

For the first time since the industrial revolution, the layman can access manufacturing tools and therefore create high quality, industry standard products. They can design and make whatever the like or have the capacity to imagine. In the past, to produce a high quality model or artifact, people had to have finely honed craft skills, be good with their hands and have an eye for detail. Now, with 3D printing and other digital making tools such as laser cutters, anyone with the computer file can produce high quality models. To create these computer files, one generally has to rely on the skills of designers or engineers, which defeats the purpose of democratization if the layman still has to rely on professionals. Therefore, the author of this paper has been working to develop another way for people to make the computer files. She designed and developed a 3D scanner based on an existing open source project to be able to scan small plasticine models. Though the scanner can be glitchy and not always work in the expected way, it does produce a representation of the model. Using this the author is exhibiting at events and conferences to create an online visual archive of what people want to 3D print. This will inform her $\mathrm{PhD}$, which seeks to find how 3D printers can be introduced into schools and other educational contexts.
The surge in the maker movement has expanded the culture that surrounds making and exposed more people to it. This is particularly important and exciting as, by empowering more people with the knowledge, belief and skills to make changes in their lives and perhaps the lives of other people, a greater majority can contribute to the design of the future, which should mean that it is more suitable to the needs, desires and values of the majority instead of the minority.

To engage the majority, there must be an appropriate platform, which laymen can interact with and use, so that they can use the technology for projects and ideas that extend beyond the gimmicky, novelty use that often involves printing a Yoda head or miniature robot. Though these are fun things to print, it is important to push the boundaries further to ensure that $3 \mathrm{D}$ printing is being used to affect social change and not to merely contribute to consumer culture.

\section{LITERATURE REVIEW}

Eisenberg (2013) highlights the similarities between the emergence of computing and the emergence of fabrication tools. In the late 70 s and 80 s, computing was where 3D printing is now. Early pioneers of computing did not fully exploit the potential and implications of computers throughout society and instead focused on business use. However, as Lipson (2013) points out, technology can only be 
picked up and appropriated by the masses through a 'killer app'. (2013:39) For computing, this perhaps only happened in the late 90 s with the Macintosh and more so in the late 00s with the iPhone and tablets. Alongside these developments in physical hardware, the advancements in networks and speed of the Internet helped the uptake of computing and enable it to have the social impact it has had since.

The maker movement provides the opportunity for $3 \mathrm{D}$ printers to be exposed and introduced to more people. Thomas (2014) discusses that things like spaceships were the dreams of children who spent their childhoods and adolescence tinkering, made these dreams a reality. She says,

'I believe that it is essential for us to empower todays children with the tools and skills they need to make their dreams tangible. The Maker Movement is a shining example of how we can do this. (2014:xii)

By ensuring that the children of today grow up thinking that they can achieve anything and make anything they can imagine, it is possible that innovations and potential social impact can arrive faster and reach further than ever before. Therefore, the Maker Movement and culture embodies and makes possible a platform on which the layman can access new digital making technologies.

\section{METHODOLOGY}

For the purposes of this pilot study, a 3D scanner designed and developed by the author from an open source project was set up at a conference as a form of conference documentation and exhibited at a mini maker faire to provide children and adults alike with the opportunities to interact with 3D scanning. The scanner was fine tuned to allow it to scan small objects. This is now being used to allow people to use plasticine to model their ideas before 3D scanning and then 3D printing them.

This technique is being used to create a visual archive of what people want to 3D print, or more broadly what they can imagine and what they want to make.

A problem, at the moment, is that $3 D$ printers, in educational contexts, have become branded as gimmicky and not the ground-breaking technology that was promised. Some schools have bought them and are attempting to use them in the classroom but at the moment, it appears that only the 'best' students are allowed to use them and that they are being used for standard, uniform designs made from step-by-step instructions. Additionally, when they break down, which is a frequent occurrence they are out of service for a relatively long period of time. Therefore, this research is trying to take a step back and work out what people want to make and what is a natural way to learn to use them. Once this becomes clear, the integration of $3 \mathrm{D}$ printers and digital making tools can be more smooth and perhaps more successful.

To attract as much participation as possible, the scans are made available through a Thingiverse collection so that individual makers are able to download their scans to have as a digital memento or to $3 \mathrm{D}$ print if they have access to a $3 \mathrm{D}$ printer.

\section{OBSERVATIONS AND ANALYSIS}

The scans, though not always or entirely representative of the models made by the participants, illustrate what the participants made with plasticine. At the conference, the delegates were asked to make a model, which represented their experience of the conference. At the Edinburgh mini maker faire, the participants were asked to make a model of something they would $3 \mathrm{D}$ print if the possibilities were endless. Due to the nature of the events, the conference participants were solely adults, while the maker faire audience was a mix.

The models produced from the conference were generally original and a logical response to the question. Whereas the models produced at the maker faire may be compared to the drawings people might do if asked to draw something on the spot, for example a smiley face or a tree in the simplistic style, a cylinder with a cloud on top.

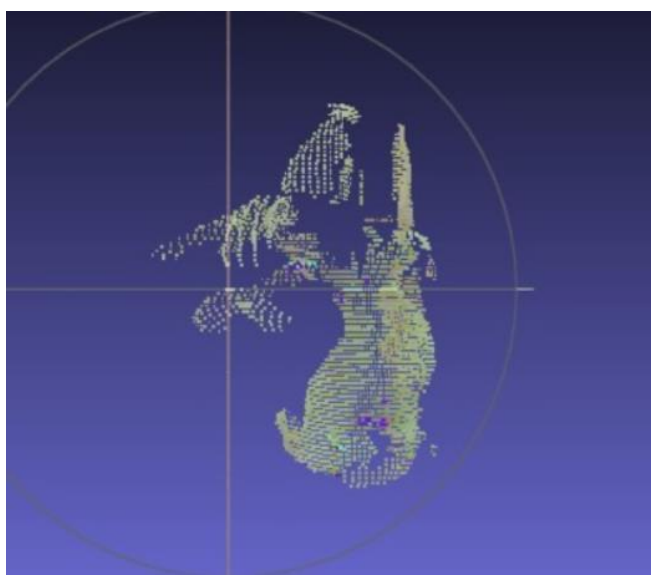

Figure 1 Scan of Plasticine Tree

Figure 1, created at the conference shows the scan of a plasticine tree made by one of the delegates. This particular delegate wrote on the card next to the model that he had made the tree as a representation of all the research coming from design. This illustrates the potential of 3D scanning as a form of expression in contexts where the 
participants may feel self-conscious about writing a standalone statement or observation, which may be considered pretentious.

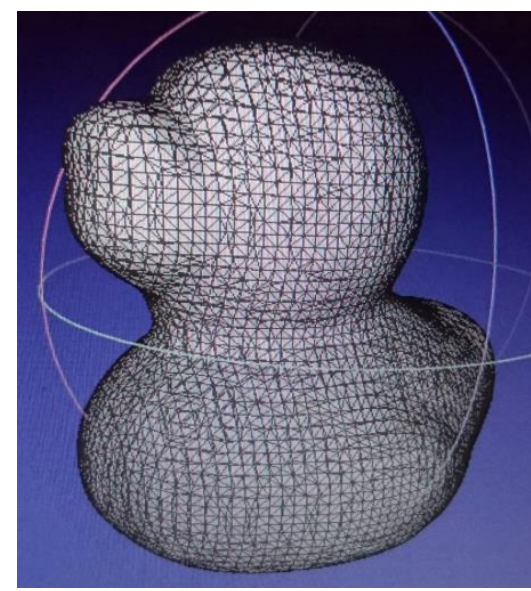

Figure 2 Scan of Plasticine Duck

In contrast, figure 2, created at the Maker Faire, shows the model produced by a child in response to the task, 'Make what you would make if you could 3D print anything.' The model does not appear to be a genuine or logical response to the question and instead perhaps just shows that the child's first response was to make what they would draw if given a pencil. Although it is possible that a child would make a duck, though it seems that given more time to imagine and become less constrained they might choose to make something other than a common bath toy. However, figure 3, created at the Maker Faire, was accompanied by a description, which explained that the model was an earpiece, which could be worn inconspicuously and the user could speak to it or ask questions before having the answers whispered back to them. Essentially it is a discreet, wearable Amazon Alexa or Siri.

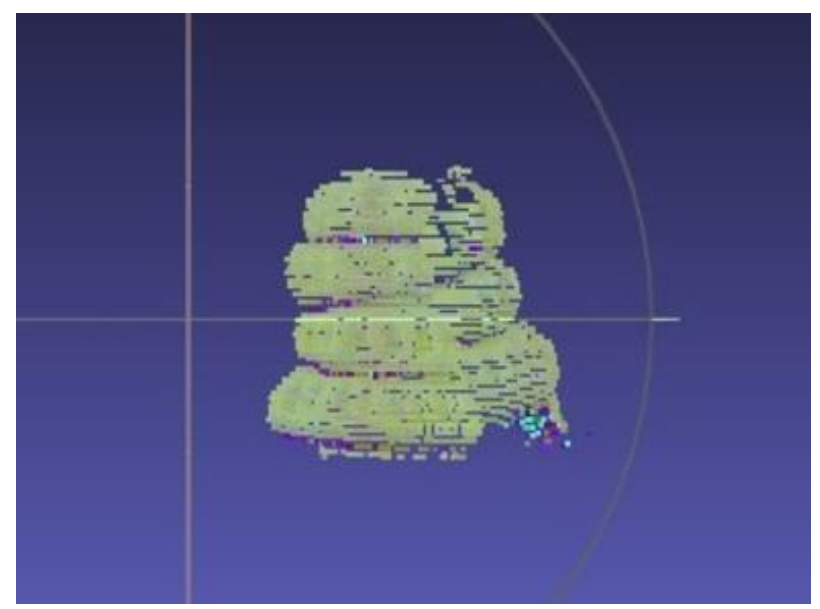

Figure 3 Scan of Plasticine Ear Piece

The data from all the scans and participant comments is yet to be fully analysed but for this short paper, the initial observations and analysis suggest that 3D scanning and quick hand modelling is an interesting and potentially useful tool in surveying or experience documentation. It is intended that the scanner will be taken to more events over the next three years to create a large online visual archive of what people want, feel, like etc. Though the analysis and findings are at a very early stage, it might be suggested that the plasticine models should be produced alongside a short description to help contextualise the idea and to clarify any components or details, which are not clear.

\section{CONCLUSION}

In conclusion, the Maker Movement is providing a unique opportunity to democratize 3D printing so that it can have a significant social impact sooner than might otherwise be possible. By using technologies such as 3D scanners and empowering the laymen to tinker with these so that they can get good results it is possible for almost anyone to be able to design and produce whatever they want or need. By doing this, innovation can occur across society and across professions. People are empowered to change their immediate environment and perhaps the lives of others.

The author is using a homemade 3D scanner to document what people want to 3D print. This serves two purposes; one being to learn more about what people expect and think is possible using a 3D printer and also to raise the profile of 3D printers as an accessible tool in contexts which might otherwise never consider 3D printing.

Scanning has a lot of negatives at the moment and will remain sub-par until a better and more affordable scanner is developed. However, just with the scanner used for this research, it is interesting to learn how different people approach 3D printing. Adults sometimes use it as a way of expressing feelings or ideas, which they may otherwise be too self-conscious to put into words, while children tend to be heavily influenced by what has already been made. However, with continued exposure and by making 3D printing not a novelty, the boundaries can be pushed and children as well as adults can begin to produce valuable and impactful ideas and concepts that might never been realised. It is early stages for this research, however, the potential of what might be discovered or created is very exciting.

\section{REFERENCES}

Eisenberg, M. (2013). 3D printing for children: What to build next?. International Journal of ChildComputer Interaction. 1 (1), p7-13. 
Lipson, H. (2013) Fabricated : the new world of 3D

printing, Indianapolis, Ind.: John Wiley \&amp; Sons.

Thomas, A. (2014) Making Makers, USA: Maker Media, inc. 